\title{
New records of ants (Hymenoptera: Formicidae) from the Chaharmahal va Bakhtiari Province of Iran with taxonomic comments
}

\author{
Arsalan Khalili-MoghadaM ${ }^{1}$, LeCH BOROWIEC $^{2 *}$, AliREZA NeMATI $^{1}$ \\ ${ }^{1}$ Plant Protection Department, Agricultural College, Shahrekord University, \\ Shahrekord, Iran \\ ${ }^{2}$ Department of Biodiversity and Evolutionary Taxonomy, University of Wrocław, \\ Przybyszewskiego, 65, 51-148 Wrocław, Poland
}

\begin{abstract}
The ant fauna from the Chaharmahal va Bakhtiari Province of Iran was surveyed. As a result, a total of 28 ant species belonging to 11 genera of Formicidae were collected and identified. Species status is proposed for Messor barbarus subsp. mediosanguineus DONISTHORPE, 1946, the species status of Messor platyceras CRAWLEY, 1920 is restored with Messor platyceras var. rubella CRAWLEY, 1920 as a new synonym of the nominotypical form, and Camponotus oasium ninivae PISARSKI, 1971 is removed from synonyms of Camponotus oasium FOREL, 1890. The genera Messor FOREL, 1890, with seven species and Cataglyphis FÖRSTER, 1850 with six species, have the highest species richness. Messor mediosanguineus DONISTHORPE, 1946, Pheidole koshewnikovi RUZSKY, 1905 and Camponotus oasium ninivae PISARSKI, 1971 were recorded in Iran for the first time.
\end{abstract}

KEY WORDS: ants, faunistics, taxonomy, new national records, new regional records, Chaharmahal va Bakhtiari Province, Iran

\section{INTRODUCTION}

Ants are among the most successful organisms on planet Earth (WILSON 1990). Knowledge of Iranian ants is relatively poor. PAKNIA et al. (2008) reviewed the literature, added new records and provided the first checklist of Iranian ant fauna. Since that time, several faunistic and taxonomic studies have been carried out in various parts of Iran (PAKNIA

\footnotetext{
*Corresponding author: lech.borowiec@uwr.edu.pl
} 
\& KAMI 2007, GHAHARI et al. 2009, 2011, 2015, RAFINEJAD et al. 2009, PAKNIA et al. 2010, RAdCHENKo \& PAKNIA 2010, FirouZI et al. 2011, MohAmmadi et al. 2012, Hossein NeZHAD et al. 2012, SHIRAN et al. 2013, HoSSEINI et al. 2015, KHANDEHROO et al. 2015, MirzAMOHAMADI et al. 2015, MoradLOO et al. 2015, MoRTAZAVI et al. 2015, GHOBADI et al. 2016, HEIDARI et al., 2017). More than 248 species have been reported from Iran to date (PASHAEI RAD et al. 2018). Considering that many areas of Iran have not been studied, the number of ant species in this country's fauna is very likely much higher. On the other hand, in view of the lack of keys for identifying the ants from this area and the fact that recent ant research from Europe and the Middle East indicates a large number of cryptic species and the distinctiveness of the West and East Mediterranean faunal complexes (CsŐsZ et al. 2015, WAGNER et al. 2017, CsŐSZ et al. 2018, SALATA et al. 2018, STEINER et al. 2018), several records from Iran are open to question and require verification or confirmation.

Chaharmahal va Bakhtiari Province is situated in the south-west of Iran on the eastern slopes of the Zagros Mountains with oak forests and grazing areas. This province has a rich and diverse flora and fauna, but the ant fauna has hardly been studied. This paper is the first one focusing on the ants of the province. Some samples remain unidentified. A preliminary overview indicates that they may contain species new to science, so comparative studies with materials from neighbouring areas will be required.

\section{MATERIALS AND METHODS}

This study focused on ant fauna and the mesostigmatid mites associated with it in Chaharmahal va Bakhtiari Province. The results of the research on mesostigmatid mites will be published separately. The specimens were collected from different counties of Chaharmahal va Bakhtiari Province during 2015-2018. For sampling purposes, not only the ants but also the soil around their nests were collected with forceps and a shovel and placed in a plastic bag. Important data - geographical location (with GPS), name of location, date, habitats, etc. - were recorded. After the samples had been transferred to the laboratory, the ants were separated using appropriate forceps and needles. The specimens were preserved in 75\% alcohol. The morphological keys by ARAKELYAN (1994), BOLTON (1994), COLLINGWOOD \& AGOSTI (1996), DLUSSKY et al. (1990) and RADCHENKO (1996b, 1998), as well as the extensive resources of comparative materials preserved in the Department of Biodiversity and Evolutionary Taxonomy, University of Wrocław, Poland, were used for identification. The specimens have been deposited in the Insect collection, Jalal Afshar Zoological Museum, Department of Plant Protection, Faculty of Agriculture, University of Tehran, Karaj, Iran, and at the Department of Biodiversity and Evolutionary Taxonomy, 
University of Wrocław. The photographs were taken using a Nikon SMZ 1500 stereomicroscope, Nikon D5200 photo camera and Helicon Focus software. All the samples were collected by the senior author and his students. An asterisk (*) indicates the first record of a taxon for the fauna of Iran. Worker caste is identified by the symbol $\Varangle$ in the text. Geographical coordinates, along with the altitude of the sampling sites, were measured in the field using GPS. Distribution data are cited mainly after BOROWIEC (2014), with corrections resulting from recent papers and work in areas not covered by this catalogue.

\section{RESULTS AND DISCUSSION}

\section{Subfamily Dolichoderinae FOREL, 1878}

1. Tapinoma FörSTER, 1850

1.1. Tapinoma erraticum (LATREILLE, 1798)

Formica erratica LATREILlE, 1798: 44; for synonyms, see BOLTON (2019).

Material examined. Bazoft region, Kachuz ( $32^{\circ} 1^{\prime} 4^{\prime} 52^{\prime \prime} \mathrm{N}, 4^{\circ} 58^{\prime} 56^{\prime \prime}$ E, 1814 $\mathrm{m}$ a.s.1.), oak forest, 8 ఛ , 02 June 2017; Ben county, Karsenak (32 $32^{\circ} 31^{\prime} 12^{\prime \prime} \mathrm{N}, 50^{\circ} 28^{\prime} 16^{\prime \prime}$ E, $2571 \mathrm{~m}$ a.s.1.), grazing area, 5 ६ , 13 July 2017; Lordegan county, Oureh (31 $31^{\circ} 03^{\prime \prime} \mathrm{N}$, 50²9' 32" E, 1756 m a.s.1.), oak forest, 11 ఛ , 11 April 2017.

Distribution. Widespread in central and southern Europe, the Caucasus, Iran, Israel, Kazakhstan, Kyrgyzstan, Turkey and Turkmenistan.

Comment. A recent paper on Albanian ants by WAGNER et al. (2018) suggested that southern populations from the Balkans recorded as Tapinoma erraticum represent a cryptic undescribed species. Parts of populations from areas east of the Balkans probably belong to the same cryptic taxon but prior to the formal description we applied the appellation $T$. erraticum to the Iranian populations.

\subsection{Tapinoma karavaievi EMERY, 1925}

Tapinoma simrothi ssp. karavaievi EMERY, 1925: 52.

Material examined. Ben county, Karsenak (32 $32^{\circ} 31^{\prime} 14^{\prime \prime} \mathrm{N}, 50^{\circ} 28^{\prime} 18^{\prime \prime}$ E, 2540 $\mathrm{m}$ a.s.1.), grazing area, 10 ६ , 13 July, 2017; Ben county, Larak (32 $35^{\prime} 14^{\prime \prime} \mathrm{N}, 50^{\circ} 40^{\prime} 27^{\prime \prime}$ E, 2425 m a.s.l.), grazing area, 7 ६ , 07 April, 2017; Boldaji region, Chamangoli $\left(32^{\circ} 16^{\prime}\right.$ $10^{\prime \prime} \mathrm{N}, 49^{\circ} 57^{\prime} 36^{\prime \prime} \mathrm{E}, 1925 \mathrm{~m}$ a.s.1.), grazing area, 6 ф , 01 June 2017; Cheghakhor (31 $51^{\prime}$ $12^{\prime \prime} \mathrm{N}, 50^{\circ} 56^{\prime} 30^{\prime \prime} \mathrm{E}, 2295 \mathrm{~m}$ a.s.l.), ant nest in poplar trees, 6 ఛ , 21 June, 2013; Farsan county (32 $12^{\prime} 41^{\prime \prime} \mathrm{N}, 50^{\circ} 37^{\prime} 30^{\prime \prime} \mathrm{E}, 2008 \mathrm{~m}$ a.s.1.), grazing area, 16 ф , 14 April, 2017; Kohrang county, Sudejan ( $32^{\circ} 32^{\prime} 25^{\prime \prime} \mathrm{N}, 50^{\circ} 21^{\prime} 08^{\prime \prime} \mathrm{E}, 2391 \mathrm{~m}$ a.s.1.), grazing area, 19 ф , 25 April, 2017; Lordegan county, Bardbor (31 $31^{\circ} 48^{\prime \prime}$ N, 50 01' 14" E, 1819 m a.s.1.), ant nest in poplar trees, 11 ६ , 21 June, 2013; Lordegan county, Oureh (31 $30^{\circ} 17^{\prime \prime} \mathrm{N}, 50^{\circ} 30^{\prime}$ 
03" E, 1753 m a.s.1.), oak forest, 23 ఛ, 14 April, 2017; Saman county, Shoorab (32 $32^{\prime}$ 40" N, 50 56' 13" E, $2015 \mathrm{~m}$ a.s.1.), ant nest in almond garden, 17 ६ , 05 May, 2016; Sheshyek farm ( $32^{\circ} 27^{\prime} 29^{\prime \prime} \mathrm{N}, 50^{\circ} 54^{\prime} 573^{\prime \prime} \mathrm{E}, 1943 \mathrm{~m}$ a.s.1.), walnut garden, 8 ㅎ, 10 June, 2013; Shahrekord county, Chaleshtor (32 ${ }^{\circ} 23^{\prime} 09^{\prime \prime} \mathrm{N}, 50^{\circ} 45^{\prime} 10^{\prime \prime}$ E, $2099 \mathrm{~m}$ a.s.1.), agricultural area, 17 ఛ, 18 August, 2017.

Distribution. Afghanistan, Armenia, Azerbaijan, Georgia, Iran, Kazakhstan, Turkmenistan.

Comment. The status of Tapinoma karavaievi EMERY, 1925 requires revision. The male genitalia are extremely similar to those of Tapinoma simrothi KRAUSSE, 1911. Perhaps T. karavaievi is the only eastern morphotype of the widespread T. simrothi. Both taxa have been recorded in Iran but only T. karavaievi probably occurs in this area.

\section{Subfamily Formicinae LATREILLE, 1809}

1. Camponotus MAYR, 1861

1.1. Camponotus armeniacus ARNOLDI 1967

Camponotus micans subsp. armeniacus ARNOLDI, 1967: 1828.

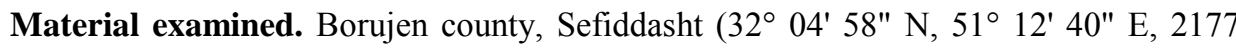
$\mathrm{m}$ a.s.1.), ant nest in a grazing area, 12 ఛ , 24 June 2014; Farokhshahr county, Tangesayad $\left(32^{\circ} 15^{\prime} 57^{\prime \prime} \mathrm{N}, 51^{\circ} 02^{\prime} 18^{\prime \prime} \mathrm{E}, 2263 \mathrm{~m}\right.$ a.s.l.), ant nest in a grazing area, 9 ㅎ , 25 June 2014.

Distribution. Armenia, Iran, Turkey.

1.2. Camponotus oasium ninivae PISARSKI, 1971*, subspecies status restored, new combination (Fig. 1)

Camponotus (Tanaemyrmex) thoracicus subsp. ninivae PISARSKI, 1971 a: 674.

Material examined. Bazoft region, Mavarz $\left(32^{\circ} 08^{\prime} 59^{\prime \prime} \mathrm{N}, 50^{\circ} 06^{\prime} 15^{\prime \prime} \mathrm{E}, 1515\right.$ m a.s.l.), oak forest, 16 ఢ , 01 June 2017.

Distribution. Iraq (PISARSKI, 1971 a); new to Iran.

Comment. RADCHENKO (1997) treated Camponotus (Tanaemyrmex) thoracicus subsp. ninivae PISARSKI, 1971 as a synonym of Camponotus oasium FOREL, 1890; this point of view was repeated in BoLTON's (2019) catalogue. In our opinion this is at least a distinct subspecies if not a good species of the Camponotus oasium species-group. This group contains a lot of different infraspecific taxa, but many of them are probably more or less cryptic species. Our sample from Mavraz agrees well with the types of $C$. oasium ninivae described from Iraq and preserved in the Museum and Institute of Zoology, Polish Academy of sciences, Warsaw, Poland (available on AntWeb CASENT0911141, CASENT0917224 and CASENT0917225). Specimens of $C$. oasium ninivae differ from the types of C. oasium oasium FOREL, 1890 from Tunisia not only in their paler colour but also 
in the softer sculpture. Thus the surface of the head in both major and minor workers
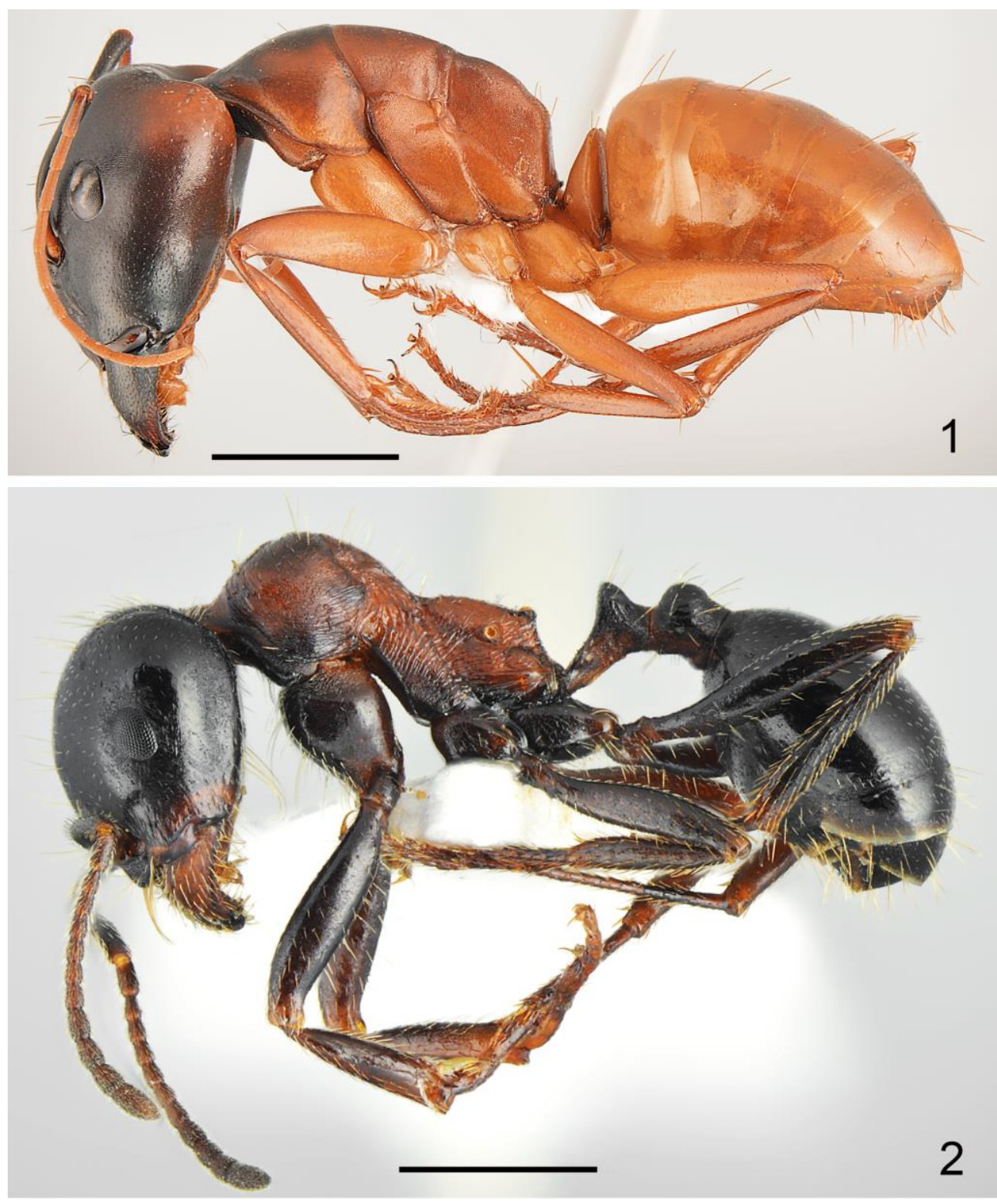

Figs 1, 2. 1. Camponotus oasium ninivae PISARSKI, 1971, major worker lateral (scale bar = $2 \mathrm{~mm}$ ); 2. Messor mediosanguineus DONISTHORPE, 1946, major worker lateral $($ scale bar = $1 \mathrm{~mm}$ ) (photo L. BOROWIEC). 
appears slightly shiny in contrast to the matt surface of the head of $C$. oasium oasium, especially in major workers. We have examined specimens similar to the $C$. oasium oasium morphotype from Fars province in Iran, but their conspecificity with true $C$. oasium is also uncertain.

\subsection{Camponotus xerxes FOREL, 1904}

Camponotus maculatus r. xerxes FOREL, 1904: 424.

Material examined. Saman county, Shoorab $\left(32^{\circ} 30^{\prime} 38^{\prime \prime}\right.$ N, 50 56 $16^{\prime \prime}$ E, 2015

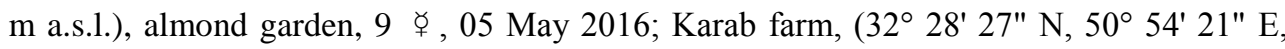
1942 m a.s.l.), walnut garden, 5 ఛ, 05 March 2014.

Distribution. Afghanistan, Egypt, Iran, Iraq, Israel, Kuwait, Turkey, Turkmenistan, Uzbekistan.

\section{Cataglyphis FöRSTER, 1850}

2.1. Cataglyphis bellicosa (KARAVAIEv, 1924)

Myrmecocystus (Cataglyphis) bicolor subsp. bellicosus KARAVAIEV, 1924: 307.

Material examined. Bazoft region, Taraz ( $32^{\circ} 21^{\prime} 07^{\prime \prime} \mathrm{N}, 49^{\circ} 52^{\prime} 46^{\prime \prime} \mathrm{E}, 1674 \mathrm{~m}$ a.s.l.), oak forest, 4 ఛ , 02 June 2017; Saman county, Shoorab (32 30' 46" N, 50 56' 06" E, 2021 m a.s.1.), almond garden, 3 ఈ, 28 March 2014.

Distribution. Iran (KARAVAIEV 1924).

\subsection{Cataglyphis foreli (RUZSKY, 1903)}

Myrmecocystus foreli RUZSKY, 1903: 36.

Material examined. Farokhshahr county, Tangesayad $\left(32^{\circ} 15^{\prime} 57^{\prime \prime} \mathrm{N}, 51^{\circ} 02^{\prime} 18^{\prime \prime} \mathrm{E}\right.$, $2263 \mathrm{~m}$ a.s.l.), ant nest in a grazing area, 5 ఢ , 25 June 2014.

Distribution. Iran, Turkmenistan.

\subsection{Cataglyphis kurdistanica PISARSKI, 1965}

Cataglyphis kurdistanicus PISARSKI, 1965: 417.

Material examined. Ben county, Karsenak (32 32' 19" N, 50 28' 07" E, 2409 m a.s.1.), grazing area, 22 ㅎ , 13 July 2017.

Distribution. Iran, Iraq, Turkey.

\subsection{Cataglyphis nigripes ARNOLDI, 1964}

Cataglyphis setipes subsp. nigripes ARNOLDI, 1964: 1806.

Material examined. Saman county, Babapir Ahmad village ( $32^{\circ} 30^{\prime} 47^{\prime \prime} \mathrm{N}, 50^{\circ} 51^{\prime} 31^{\prime \prime}$ E, $1882 \mathrm{~m}$ a.s.l.), Chelevan village ( $32^{\circ} 29^{\prime} 25^{\prime \prime} \mathrm{N}, 50^{\circ} 54^{\prime} 40^{\prime \prime} \mathrm{E}, 1884 \mathrm{~m}$ a.s.l.), walnut

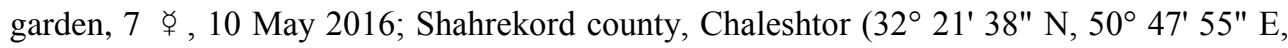


2070 m a.s.1.), agricultural area, 11 ф , 02 June 2016; Shahre-Kian, Bahram Abad (32 $15^{\prime}$ 49" N, 50 52' 15" E, 2046 m a.s.1.), agricultural area, 4 ఛ , 05 August 2015.

Distribution. Armenia, Georgia, Iran.

\subsection{Cataglyphis nodus (BRULLÉ, 1833)}

Formica nodus BRULLÉ, 1833: 326.

Material examined. Shahrekord, Shahrekord University $\left(32^{\circ} 21^{\prime} 10^{\prime \prime} \mathrm{N}, 50^{\circ} 49^{\prime} 43^{\prime \prime} \mathrm{E}\right.$, $2093 \mathrm{~m}$ a.s.1.), park area, 7 ६ , 10 May 2017; Chaleshtor $\left(32^{\circ} 21^{\prime} 46^{\prime \prime} \mathrm{N}, 50^{\circ} 45^{\prime} 56^{\prime \prime} \mathrm{E}\right.$, $2104 \mathrm{~m}$ a.s.l.), agricultural area, 11 ६ , 18 August 2017; Farokhshahr, Khyr Abad (32 $09^{\prime}$ 47" N, 50 57' 23" E, 2167 m a.s.1.), grazing area, 5 ఛ , 10 August 2017; Kiar County,

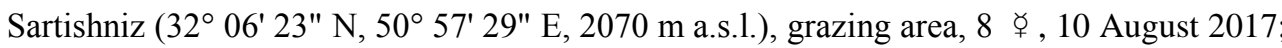
Kiar County, Dareh Garm ( $32^{\circ} 08^{\prime} 11^{\prime \prime} \mathrm{N}, 50^{\circ} 53^{\prime}$ 58" E, $2073 \mathrm{~m}$ a.s.1.), grazing area, 15 ఢ , 10 August 2017; Bazoft, Mavarz (32 08' 51" N, 50 06' 20" E, 1515 m a.s.1.); Chamangoli (32 $12^{\circ} 57^{\prime \prime} \mathrm{N}, 50^{\circ} 00^{\prime} 52^{\prime \prime}$ E, 1567 m a.s.l.), oak forest, 9 ф̧, 02 June 2017.

Distribution. Afghanistan, Albania, Arabian Peninsula, Armenia, Azerbaijan, Bulgaria, Bosnia and Herzegovina, Croatia, Georgia, Greece, Egypt, Hungary, Iran, Israel, Iraq, Montenegro, North Macedonia, Romania, Russia (North Caucasus), Serbia, Slovakia, Syria, Turkey, Turkmenistan, Uzbekistan.

Comment. The Iranian samples are generally darker in colour than the typical populations from the Balkans. Unfortunately, the material from Chaharmahal va Bakhtiari Province contained only workers, so we were unable to compare the male genitalia of the Iranian and Balkan populations. We do not know, therefore, whether these darker populations represent a separate morphotype or even a separate species.

\subsection{Cataglyphis setipes (FOREL, 1894)}

Myrmecocystus viaticus $\mathrm{r}$. setipes FOREL, 1894: 60.

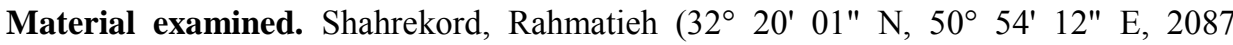
$\mathrm{m}$ a.s.1.), ant nest in an agricultural area, 5 ఢ , 18 August 2017; Saman county, Shoorab ( $32^{\circ} 17^{\prime} 29^{\prime \prime} \mathrm{N}, 50^{\circ} 49^{\prime} 08^{\prime \prime}$ E, $2054 \mathrm{~m}$ a.s.1.), almond garden, 11 ఢ̧, 02 June 2016.

Distribution. Afghanistan, Dagestan, Iran, Kazakhstan, Kyrgyzstan, Turkey, Turkmenistan, Uzbekistan.

\section{Formica LINNAEUS, 1758}

3.1. Formica clara FOREL, 1886 sensu SEIFERT \& SCHUltz (2009)

Formica rufibarbis var. clara FOREL, 1886: 206; for synonyms, see BOROWIEC 2014: 73.

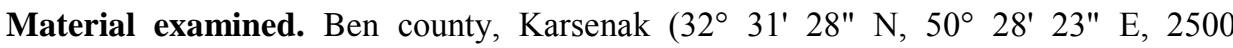
m a.s.1.), grazing area, 5 ఢ, 13 July 2017. 
Distribution. Central and southern Europe, Azerbaijan, China, Cyprus, Georgia, northern India, Iran, Israel, Kazakhstan, Kyrgyzstan, Pakistan, Syria, Turkey, Turkmenistan (SEIFERT \& SCHULZ 2009).

\section{Lasius FABRICIUS, 1804}

4.1. Lasius neglectus VAN LOON, BOOMSMa \& ANDRASFALVY, 1990

Lasius (Lasius) neglectus VAN LOON, BoOMSMA \& ANDRASFALVY, 1990: 350.

Material examined. Saman county, Kahkesh $\left(32^{\circ} 28^{\prime} 45^{\prime \prime} \mathrm{N}, 50^{\circ} 55^{\prime} 10^{\prime \prime}\right.$ E, $1839 \mathrm{~m}$

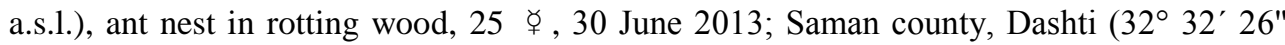

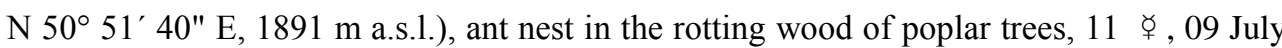
2013; Saman county, Markadeh ( $32^{\circ} 40^{\prime} 49^{\prime \prime}$ N, 50 48' 59" E, 1952 m a.s.l.), walnut garden, 9 ㅎ , 31 July 2017; Saman county, Gharagoosh $\left(32^{\circ} 31^{\prime} 39^{\prime \prime} \mathrm{N}, 50^{\circ} 48^{\prime} 06^{\prime \prime}\right.$ E, $1965 \mathrm{~m}$ a.s.l.), ant nest in the rotting wood of poplar trees, 15 ఛ, 31 July 2017; Saman

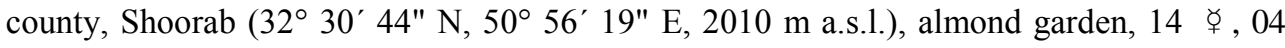
June 2013; Saman county, Chelevan ( $32^{\circ} 28^{\prime} 46^{\prime \prime}$ N, 50 55' 21" E, $1840 \mathrm{~m}$ a.s.l.), ant nest

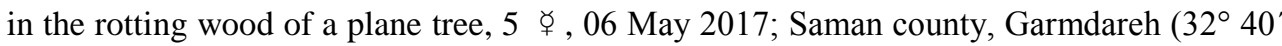
$20^{\prime \prime} \mathrm{N}, 50^{\circ} 49^{\prime} 021 " \mathrm{E}$, $1953 \mathrm{~m}$ a.s.l.), walnut garden, 30 㝏, 31 July 2017; Ben county, Bardeh ( $32^{\circ} 33^{\prime} 46^{\prime \prime} \mathrm{N}, 50^{\circ} 32^{\prime} 04^{\prime \prime}$ E, 2379 m a.s.l.), ant nest in the rotting wood of willow

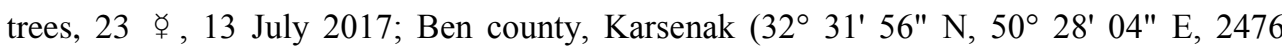
m a.s.l.), grazing area, 9 ;, 13 July 2017; Nafch $\left(32^{\circ} 23^{\prime} 52^{\prime \prime} \mathrm{N}, 50^{\circ} 47^{\prime} 17^{\prime \prime}\right.$ E, 2095 $\mathrm{m}$ a.s.1.), ant nest in the rotting wood of willow trees, 28 ; , 16 Jun 2017.

Distribution. Andorra, Belgium, Bulgaria, England, France, Georgia, Germany, Greece, Hungary, Iran, Israel, Italy, Kyrgyzstan, Macaronesia, the Netherlands, Poland, Portugal, Romania, Russia (European part), Spain, Switzerland, Turkey, Ukraine, Uzbekistan.

\section{Lepisiota SANTSCHI, 1926}

\subsection{Lepisiota karawaiewi (KUZNETSOV-UGAMSKY, 1929)}

Acantholepis frauenfeldi subsp. karawaiewi KUZNETSOv-UGAMSKY, 1929a: 483 (=Acantholepis frauenfeldi melas var. karawaiewi SANTSCHI, 1917: 44 unavailable name).

Material examined. Samsami region, Chery mountain $\left(32^{\circ} 10^{\prime} 04^{\prime \prime} \mathrm{N}, 50^{\circ} 10^{\prime} 04^{\prime \prime} \mathrm{E}\right.$, $2811 \mathrm{~m}$ a.s.1.), mountain area, $7 \quad \Varangle, 06$ July 2017.

Distribution. Iran, Kazakhstan, Kuwait, Uzbekistan.

\subsection{Lepisiota semenovi (RUZSKY, 1905)}

Acantholepis frauenfeldi var. semenovi RUZSKY, 1905: 461; for synonyms, see BOROWIEC 2014: 95. 
Material examined. Bazoft region, Sartang Sardab (31 ${ }^{\circ} 59^{\prime} 40^{\prime \prime} \mathrm{N}, 50^{\circ} 53^{\prime} 39^{\prime \prime} \mathrm{E}, 1760$ m a.s.1.), oak forest, 23 ఢ , 02 June 2017; Kian county, Bahramabad (32 $15^{\circ} 47^{\prime \prime} \mathrm{N}, 50^{\circ} 51^{\prime}$ 09" E, $2047 \mathrm{~m}$ a.s.1.), ant nest in an agricultural area, 4 ఢ , 05 August 2015; Borujen county, Sefiddasht ( $32^{\circ} 04^{\prime} 58^{\prime \prime} \mathrm{N}, 51^{\circ} 12^{\prime} 40^{\prime \prime} \mathrm{E}, 2177 \mathrm{~m}$ a.s.1.), ant nest in a grazing area, 3 ఛ , 24 June 2014; Farokhshahr county, Tangesayad (32 $12^{\circ}$ 57" N, 51 $02^{\prime} 18^{\prime \prime}$ E, 2263 $\mathrm{m}$ a.s.1.), ant nest in a grazing area, 7 ఢ , 25 June 2014.

Distribution. Iran, Kazakhstan, Kyrgyzstan, Russia (North Caucasus), Turkmenistan.

\section{Plagiolepis MAYR, 1861}

6.1. Plagiolepis perperamus SALATA, BOROWIEC \& RADCHENKO, 2018

Plagiolepis perperamus SALATA, BOROWIEC \& RADCHENKO, 2018: 816 (= Plagiolepis pallescens sensu RADCHENKO 1996a: 184).

Material examined. Samsami region, Chery mountain $\left(32^{\circ} 09^{\prime} 49^{\prime \prime} \mathrm{N}, 50^{\circ} 16^{\prime} 33^{\prime \prime} \mathrm{E}\right.$, 2028 m a.s.1.), walnut garden, 9 ф , 02 June 2017.

Distribution. Greece, Iran, North Macedonia, Turkey (SALATA et al. 2018).

\subsection{Plagiolepis pallescens FOREL, 1889}

Plagiolepis pallescens FOREL, 1889: 265 (= Plagiolepis tauricus sensu RADCHENKO 1996a: 185); for synonyms see SALATA et al. 2018: 812.

Material examined. Shahrekord, Chaleshtor $\left(32^{\circ} 21^{\prime} 56^{\prime \prime} \mathrm{N}, 50^{\circ} 45^{\prime} 48^{\prime \prime}\right.$ E, 2104 m a.s.1.), agricultural area, 11 ф , 18 August 2017.

Distribution. Albania, Armenia, Austria, Belgium, Bosnia and Herzegovina, Bulgaria, China, Croatia, Czech Republic, France, Germany, Greece, Hungary, Iran, Italy, Kyrgyzstan, Libya, Montenegro, North Korea, North Macedonia, Romania, Russia (European part), Serbia, Slovakia, Slovenia, Spain, Switzerland, Turkey, Ukraine, United Kingdom.

\section{Subfamily Myrmicinae FOREL, 1878 \\ 1. Crematogaster LUND, 1831}

\subsection{Crematogaster sorokini RUZSKY, 1905}

Crematogaster auberti var. sorokini RUZSKY, 1905: 495.

Material examined. Shahrekord county, Chaleshtor (32 $22^{\circ} 38^{\prime \prime} \mathrm{N}, 50^{\circ} 47^{\prime} 55^{\prime \prime}$ E, 2070 $\mathrm{m}$ a.s.1.), ant nest in an agricultural area, 7 ф̧ , 02 June, 2016.

Distribution. Iran, Iraq, Kazakhstan, Turkmenistan.

\subsection{Crematogaster subdentata MAYR, 1877}

Crematogaster subdentata MAYR, 1877: 19; for synonyms, see BOROWIEC, 2014: 69. 
Material examined. Saman county, Gharagoosh $\left(32^{\circ} 41^{\prime} 55^{\prime \prime} \mathrm{N}, 50^{\circ} 48^{\prime} 17^{\prime \prime} \mathrm{E}, 1959\right.$ $\mathrm{m}$ a.s.1.), ant nest in rotting wood, 7 ఛ , 31 July 2017; Saman county, Markadeh $\left(32^{\circ} 40^{\prime}\right.$

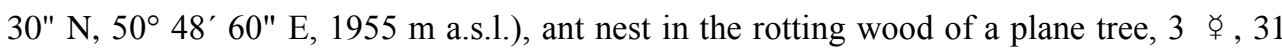

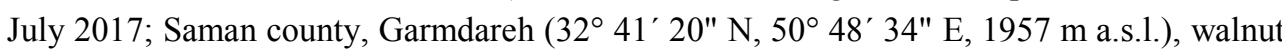
garden, 5 ఛ , 31 July 2017.

Distribution. Afghanistan, Armenia, western China, Georgia, Iran, Kazakhstan, Kyrgyzstan, Mongolia, Turkmenistan, Russia, Ukraine, Uzbekistan.

\section{Messor FOREL, 1890}

2.1. Messor caducus (MoTSCHOULSKY 1839)

Formica caduca MOTSCHOULSKY, 1839: 47.

Material examined. Saman county, Shoorab $\left(32^{\circ} 30^{\prime} 38^{\prime \prime}\right.$ N, 50 56 $56^{\prime} 13^{\prime \prime}$ E, 2015 m a.s.l.), almond garden, 8 ఛ , 05 May 2016; Kian county, Bahram Abad (32 $15^{\prime} 47^{\prime \prime} \mathrm{N}$, $50^{\circ} 51^{\prime} 39^{\prime \prime} \mathrm{E}, 2047 \mathrm{~m}$ a.s.l.), agricultural area, 9 ६, 30 July 2016; Bazoft region, Chamangoli $\left(32^{\circ} 12^{\prime} 53^{\prime \prime} \mathrm{N}, 50^{\circ} 00^{\prime} 45^{\prime \prime} \mathrm{E}, 1616 \mathrm{~m}\right.$ a.s.l.), grazing area, 17 ఈ, 02 June 2017.

Distribution. Armenia, Georgia, Iran, Kazakhstan, Turkey.

\subsection{Messor incorruptus KUZNETSOV-UgAMSKY, 1929}

Messor barbarus subsp. incorruptus KUZNETSOV-UGAMSKY 1929 b: 16 (= Messor barbarus ssp. aegyptiacus var. incorruptus RUZSKY, 1905: 751 unavailable name).

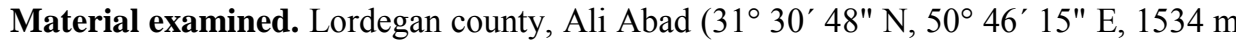
a.s.l.), ant nest in an agricultural area, 5 ఛ , 24 March 2014; Farsan county, Gusheh (32 $12^{\prime} 18^{\prime \prime} \mathrm{N}, 50^{\circ} 35^{\prime} 53^{\prime \prime} \mathrm{E}, 2005 \mathrm{~m}$ a.s.l.), ant nest in an agricultural area, 3 ㅎ, 10 September 2016; Hafshejan county, Cheshmehzaneh ( $32^{\circ} 12^{\prime} 17^{\prime \prime} \mathrm{N}, 50^{\circ} 46^{\prime} 03^{\prime \prime} \mathrm{E}, 2120 \mathrm{~m}$ a.s.l.), ant nest in a grazing area, 9 ఛ, 06 June 2015; Farokhshahr county, Tangesayad (32 $15^{\prime} 57^{\prime \prime} \mathrm{N}$, $51^{\circ} 02^{\prime} 18^{\prime \prime}$ E, $2263 \mathrm{~m}$ a.s.l.), ant nest in a grazing area, 8 ; , 25 June 2014; Kiar county, Irancheh ( $32^{\circ} 07^{\prime} 15^{\prime \prime} \mathrm{N}, 50^{\circ} 03^{\prime} 31^{\prime \prime}$ E, $2247 \mathrm{~m}$ a.s.l.), ant nest in the Tangesayad National Park, 6 ㅎ , 24 June 2014.

Distribution. Armenia, Georgia, Iran, Kazakhstan, Lebanon, Syria.

\subsection{Messor intermedius SANTSCHI, 1927}

Messor semirufus var. intermedius SANTSCHI, 1927: 229 (= Messor barbarus ssp. semirufus var. intermedia FOREL, 1910: 10 unavailable name).

Material examined. Borujen county, Sefiddasht ( $32^{\circ} 04^{\prime} 58^{\prime \prime} \mathrm{N}, 51^{\circ} 12^{\prime} 40^{\prime \prime} \mathrm{E}, 2177$ $\mathrm{m}$ a.s.l.), ant nest in a grazing area, 58 市, 24 June 2014.

Distribution. Iran, Israel, Syria, Turkey, Turkmenistan. 


\subsection{Messor mediosanguineus DONISTHORPE, 1946* new status (Fig. 2)}

Messor barbarus subsp. mediosanguineus DONISTHORPE, 1946: 53.

Material examined. Bazoft region, Deh Chendar (32 14' 50" N, 49 59' 09" E, 1774 $\mathrm{m}$ a.s.1.), ant nest in a grazing area, 30 ఛ , 02 June 2017; Ben county, Larak (32 $35^{\prime} 12^{\prime \prime} \mathrm{N}$, $50^{\circ} 40^{\prime} 22^{\prime \prime}$ E, $2046 \mathrm{~m}$ a.s.1.), ant nest in a grazing area, 7 ф , 07 April 2017; Farokhshahr county, Tangesayad ( $32^{\circ} 16^{\prime} 28^{\prime \prime} \mathrm{N}, 51^{\circ} 01^{\prime} 20^{\prime \prime} \mathrm{E}, 2365 \mathrm{~m}$ a.s.1.), ant nest in a grazing area, 6 ఛ , 24 June 2014.

Distribution. Turkey (DONISTHORPE 1946); new to Iran.

Comment. This is the first certain record of $M$. mediosanguineus from Iran. A previous record of Messor denticulatus SANTSCHI, 1927 (now a junior synonym of Messor laboriosus SANTSCHI, 1927) from western Iran (PASHAEI RAD et al. 2018) probably concerns $M$. mediosanguineus. M. laboriosus is a species with a more easterly distribution, widespread in Central Asia, and only the records from NE Iran are reliable. $M$. mediosanguineus is a more westerly species hitherto known only from Turkey. After studying the paratype preserved in the Museum of Natural History, London (available on AntWeb, specimen no. CASENT0900484), we concluded that it is a good species, not a subspecies of Messor barbarus (LINNAEUS, 1767). Messor barbarus is distributed in the western part of the Mediterranean Basin east to Italy. It has long and dense, erect setae on the first gastral tergite and usually a red head (but some specimens have the head partly or completely black, or the whole body black) and a regularly rounded propodeum, whereas $M$. mediosanguineus has the head black, the mesosoma mostly reddish, the propodeum with two obtuse denticles and the first gastral tergite with only a few short, erect setae. Messor laboriosus differs from M. mediosanguineus in the more setose first gastral tergite and the reddish-brown head (black in M. mediosanguineus) and the yellowish to yellowish-brown legs (mostly black in M. mediosanguineus). Messor mediosanguineus belongs to the complex of species close to Messor semirufus (ANDRÉ, 1883), very speciose and diverse in the Middle East, and requiring revision owing to the number of infraspecific names available in the nomenclature.

\subsection{Messor platyceras CRAWLEY, 1920 restored species status (Figs 3-9)}

Messor platyceras CRAWLEY, 1920: 163; EMERY, 1921: 210 (as a subspecies of Messor structor (LATREILLE, 1798).

Messor platyceras var. rubella CRAWLEY, 1920: 164, new synonymy.

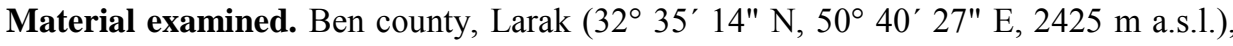
nest in the soil in a grazing area, 23 ఛ , 7 and 11 April 2017; Ben county, Karsenak $\left(32^{\circ} 31^{\prime}\right.$ $16^{\prime \prime} \mathrm{N}, 50^{\circ} 28^{\prime} 23^{\prime \prime}$ E, $2528 \mathrm{~m}$ a.s.1.), ant nest in the soil on an alfafa farm, 11 ఢ , 13 July 2017; Kohrang county, Bazoft (32 $12^{\circ} 53^{\prime \prime}-32^{\circ} 28^{\prime} 18^{\prime \prime} \mathrm{N}, 49^{\circ} 45^{\prime} 53^{\prime \prime}-50^{\circ} 00^{\prime} 45^{\prime \prime} \mathrm{E}$, 


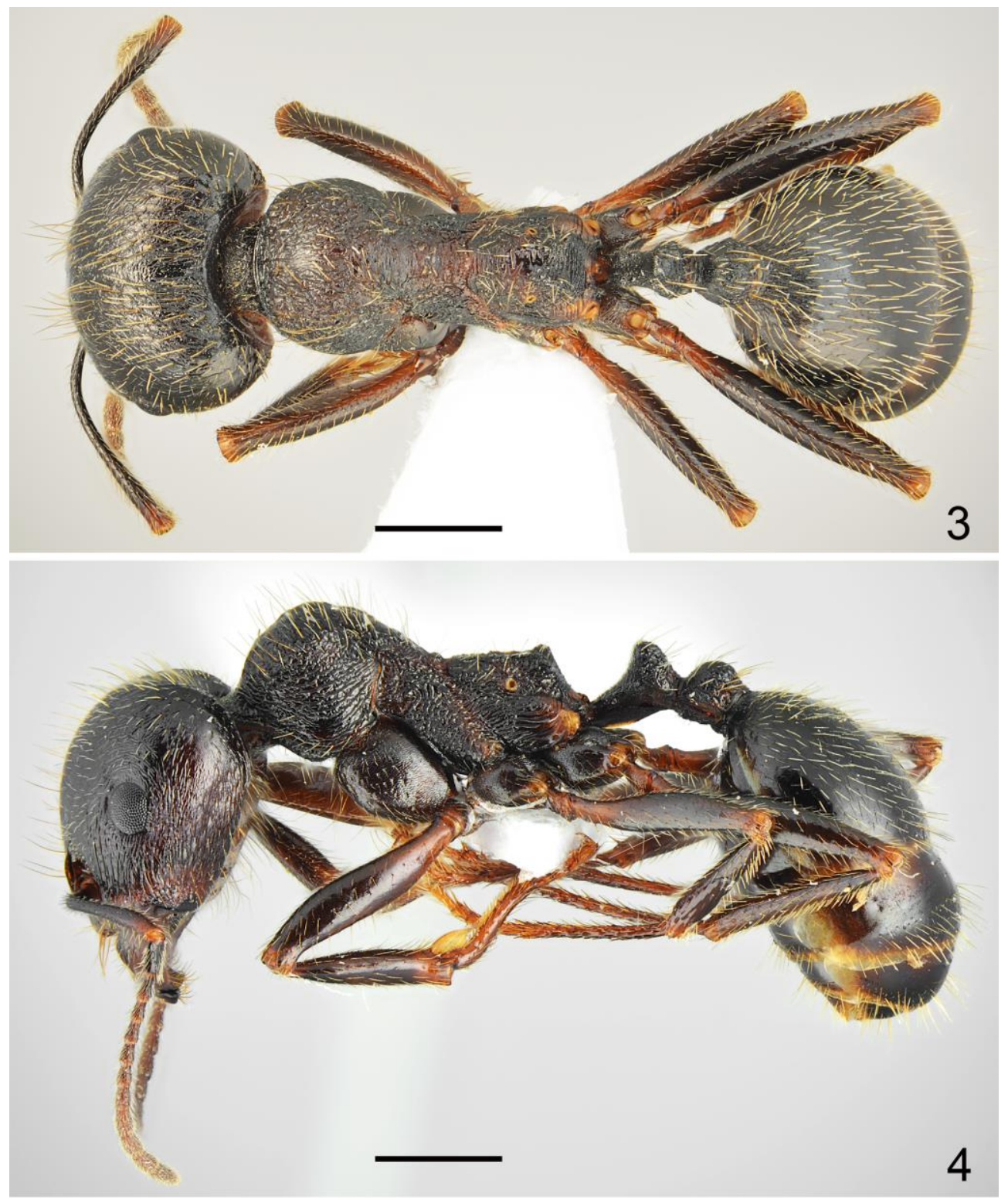

Figs 3, 4. Messor platyceras CRAWLEY, 1920: 3. Major worker dorsal, 4. Major worker lateral (scale bar $=1 \mathrm{~mm}$ ) (photo L. BOROWIEC). 

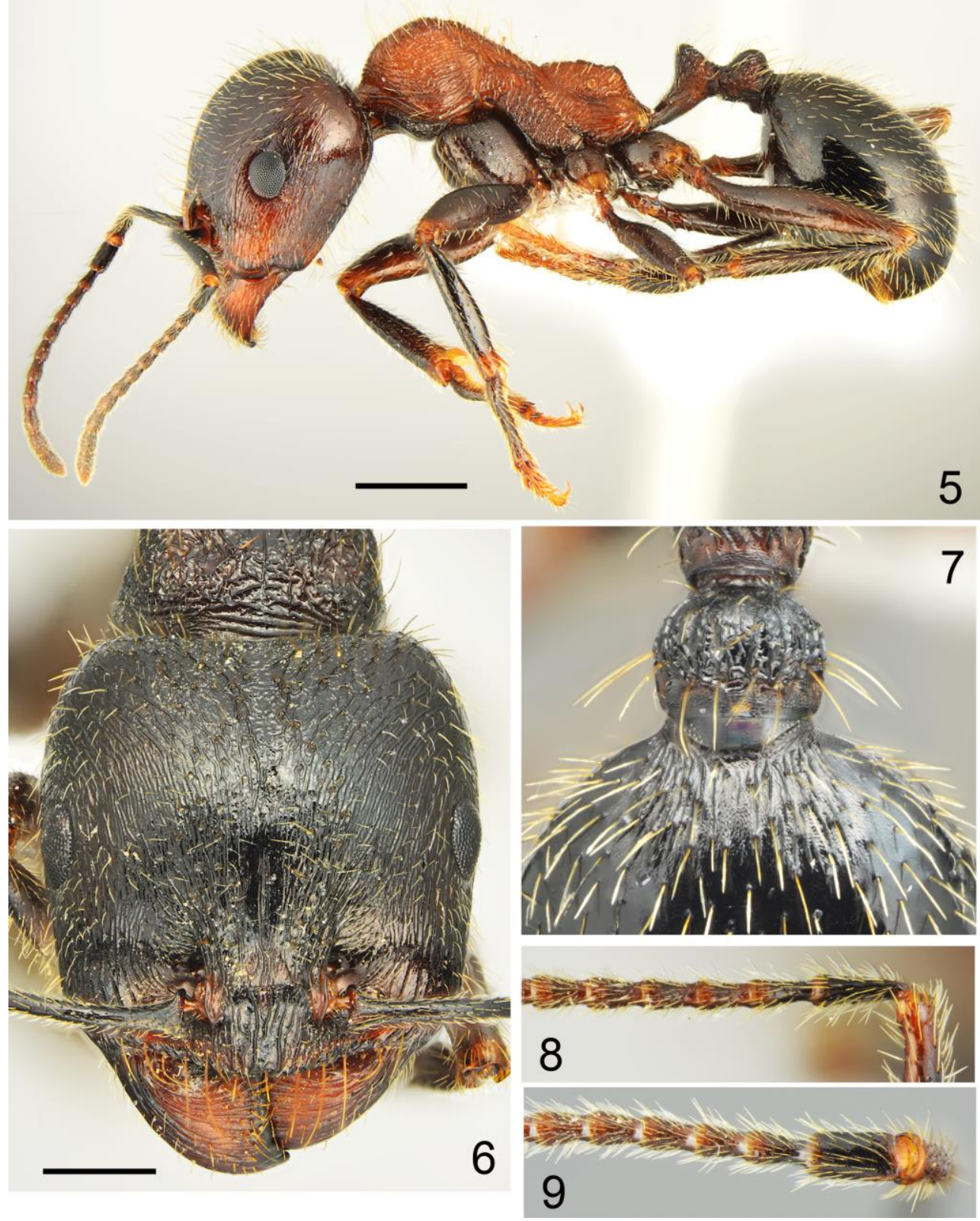

Figs 5-9. Messor platyceras CRAWLEY, 1920: 5. Major worker of ab. rubella lateral (scale bar $=1 \mathrm{~mm}$ ), 6. Head (scale bar $=0.5 \mathrm{~mm})$, 7. Base of first gastral tergite, 8. Basal segments of funiculus in anterior view, 9. Basal segments of funiculus in lateral view (photo L. BOROWIEC). 
1613 - $1942 \mathrm{~m}$ a.s.l. (23 sampling points)), ant nest in the soil in a grazing area and in an oak forest, 55 ㅁ , 1-2 June 2017; Kohrang county, Dejdaran valley (32 $11^{\circ} 44^{\prime \prime} \mathrm{N}, 50^{\circ} 12^{\prime}$ 27" E, 2319 m a.s.l.), nest in the soil in a grazing area, 9 ㅎ , 2 June 2017; Kohrang county, Soodejan ( $32^{\circ} 32^{\prime} 33^{\prime \prime} \mathrm{N}, 50^{\circ} 21^{\prime} 02^{\prime \prime} \mathrm{E}, 2143 \mathrm{~m}$ a.s.l.), nest in the soil in a grazing area and on a wheat farm, 27 ఈ , 24 March 2017, 25 May 2017 and 25 April 2017; Kiar county, Sartishniz (32 $06^{\prime} 03^{\prime \prime} \mathrm{N}, 50^{\circ} 51^{\prime} 38^{\prime \prime}$ E, $2011 \mathrm{~m}$ a.s.l.), ant nest in the soil in a grazing

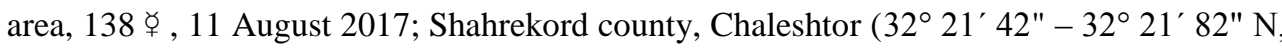
$50^{\circ} 45^{\prime} 25^{\prime \prime}-50^{\circ} 45^{\prime} 56^{\prime \prime}$ E, $2090-2104 \mathrm{~m}$ a.s.l. (7 sampling points)), ant nest in the soil in an agricultural area, 43 \% , 18 August 2017; Shahrekord county, Nafch (31 $23^{\circ} 59^{\prime \prime} \mathrm{N}$, $50^{\circ} 47^{\prime} 18^{\prime \prime}$ E, $2094 \mathrm{~m}$ a.s.l.), ant nest in the soil in a grazing area, 21 支, 18 August 2017.

Distribution. Both the nominotypical taxon and its variety were described from NW Persia without the precise locality being stated.

Comment: Based solely on the original description, EMERY (1921) placed Messor platyceras CRAWLEY, 1920 as a subspecies of Messor structor (LATREILlE, 1798). This point of view was accepted by BOLTON (1995). In their revision of the Messor structor group, STEINER et al. (2018) commented regarding M. platyceras and its variety that both taxa "do not fit morphologically either of the two species occurring closest to Iran, $M$. muticus and Lineage 7." and concluded that both of CRAWLEY's taxa were "not relevant to identifying names for any of the species characterized here". The location of the types is unknown, but CRAWLEY (1920) well characterized M. platyceras by the following combination of characters: first segment of funiculus longer than $2^{\text {nd }}$, flattened so as to be twice as long as broad with parallel sides, and much wider than $2^{\text {nd }}$ segment when viewed on its flat side (Figs 8, 9), strong body sculpture (Figs 3-6), and base of gaster longitudinally striate for a short distance, the rest smooth and shining (Fig. 7). This combination of characters is unique within the Messor structor group, especially the distinctly striate base of the gaster in combination with the elongate and flattened first segment of the funiculus. This set of characters was observed in several of the samples noted above, and we concluded that they belong to this forgotten taxon. The location of the types of M. platyceras is unknown, but the type locality (NW Iran) and the unique set of characters led us to infer that Messor platyceras is a good species from the Messor structor group. The samples examined indicated that there were workers in a single nest with an almost completely black body (Fig. 4) and with a partly reddish mesosoma (Fig. 5). We therefore concluded that Messor platyceras var. rubella was only an infraspecific colour variation. 


\subsection{Messor syriacus TOHMÉ 1969}

Messor syriacus TOHMÉ 1969: 9 (= Messor minor st. laborious var. syriacus SANTSCHI, 1927: 241 unavailable name).

Material examined. Saman county, Karab farm (32 $32^{\circ} 27^{\prime} 42^{\prime \prime} \mathrm{N}, 50^{\circ} 54^{\prime} 30^{\prime \prime}$ E, 1953 m a.s.1.), ant nest in a walnut garden, 25 ६ , 29 June 2017; Nafch $\left(32^{\circ} 23^{\prime} 52^{\prime \prime} \mathrm{N}, 50^{\circ} 47^{\prime}\right.$ 17" E, $2095 \mathrm{~m}$ a.s.1.), ant nest in the rotting wood of willow trees, 17 ఛ, 16 June 2017; Farokhshahr county, Kheyrabad ( $32^{\circ} 08^{\prime} 09^{\prime \prime} \mathrm{N}, 50^{\circ} 55^{\prime} 49^{\prime \prime} \mathrm{E}, 2073 \mathrm{~m}$ a.s.1.), ant nest in the soil in an agricultural area 13 ६ , 12 May 2017.

Distribution. Armenia, Iran, Iraq, Israel, Lebanon, Syria, Turkey.

\subsection{Messor variabilis KUZNETSOV-UGAMSKY, 1927}

Messor variabilis KUZNETSOV-UGAMSKY, 1927: 94; Messor excursionis var. macrops SANTSCHI, 1927: 248.

Material examined. Farokhshahr county, Tangesayad $\left(32^{\circ} 16^{\prime} 28^{\prime \prime} \mathrm{N}, 51^{\circ} 01^{\prime} 20^{\prime \prime} \mathrm{E}\right.$, $2365 \mathrm{~m}$ a.s.1.), ant nest in a grazing area, 5 ఛ , 24 June 2014.

Distribution. Iran, Kazakhstan, Turkmenistan.

\section{Monomorium MAYR, 1855}

\subsection{Monomorium abeillei ANDRE, 1881}

Monomorium abeillei ANDRÉ, 1881: 531.

Material examined. Cholicheh region, Rastab (32 $12^{\circ}$ '44" N, 50 36' 55" E, 2011 $\mathrm{m}$ a.s.1.), ant nest in an agricultural area, 14 ఢ, 14 April 2017; Shahrekord county,

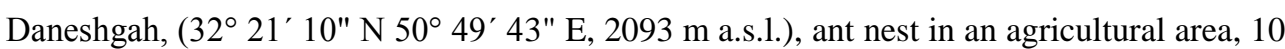
६ , 10 May 2015; Gahroo region ( $32^{\circ} 00^{\prime} 19^{\prime \prime} \mathrm{N}, 50^{\circ} 52^{\prime} 08^{\prime \prime} \mathrm{E}, 2098 \mathrm{~m}$ a.s.l.), ant nest in an agricultural area, 7 ф , 10 May 2017.

Distribution. Afghanistan, Egypt, Iran, Israel, Kuwait.

\section{Pheidole WESTWOOD, 1839}

\subsection{Pheidole koshewnikovi RUZSKY, 1905*}

Pheidole pallidula subsp. koshewnikovi RUZSKY, 1905: 648

Material examined. Saman county, Markadeh $\left(32^{\circ} 40^{\prime} 20^{\prime \prime}\right.$ N, 50 49' 02" E, 1946 $\mathrm{m}$ a.s.1.), ant nest in a walnut garden, 40 ఢ, 06 July 2017; Samsami region, Chery mountain ( $32^{\circ} 11^{\prime} 16^{\prime \prime} \mathrm{N}, 50^{\circ} 11^{\prime} 58^{\prime \prime} \mathrm{E}, 2389 \mathrm{~m}$ a.s.1.), ant nest in a grazing area, 5 ф, 06 July 2017; Bazoft region, Deh Chendar (32 $14^{\prime} 45^{\prime \prime} \mathrm{N}, 49^{\circ} 59^{\prime} 09^{\prime \prime} \mathrm{E}, 1775 \mathrm{~m}$ a.s.1.), ant nest in a grazing area, 9 ㅎ , 01 April 2016; Mavarz (32 $08^{\prime} 59^{\prime \prime} \mathrm{N}, 50^{\circ} 06^{\prime} 15^{\prime \prime} \mathrm{E}, 1515$ $\mathrm{m}$ a.s.1.), ant nest in an oak forest, 7 ఛ , 01 June 2017; Ben county, Karsenak (32 $31^{\prime}{ }^{\prime} 15^{\prime \prime}$ $\mathrm{N}, 50^{\circ} 28^{\prime} 25^{\prime \prime}$ E, $2531 \mathrm{~m}$ a.s.1.), ant nest in a grazing area, 6 ఛ , 13 July 2017. 
Distribution. Azerbaijan, Cyprus, Greece, Kazakhstan, Kyrgyzstan, Montenegro, Russia (south of European part), Turkey, Uzbekistan (SEIFERT 2016).

Comment. This is the first record of $P$. koshewnikovi from Iran. According to the recent revision of the Pheidole pallidula species-group (SEIFERT 2016), however, all previous Iranian records of Pheidole pallidula probably relate to P. koshewnikovi, which is the species with the easternmost distribution of this group.

\section{ACKNOWLEDGEMENTS}

We greatly appreciate the logistical support provided by the Plant Protection Department, College of Agriculture, Shahrekord University, Shahrekord, Iran, and all the students who helped us with the field sampling during this research. Thanks also go to Dr A. RADCHENKO (Kiev, Ukraine) for identifying some Cataglyphis and Messor species, and to two anonymous reviewers for their valuable comments.

\section{REFERENCES}

ANDRÉ E. 1881. [Untitled. Monomorium Abeillei, n. sp.]. p. 531 in: C EMERY.. Viaggio ad Assab nei Mar Rosso dei Signori G. Doria ed O. BECCARI con il R. Avviso "Esploratore" dal 16 Novembre 1879 al 26 Febbraio 1880. I. Formiche. Annali del Museo Civico di Storia Naturale, 16: 525-535.

Arakelyan G.R. 1994. Fauna of Republic of Armenia. Hymenopterous Insects. Ants (Formicidae). Armenian Academy of Sciences, Erevan.

ARNOLDI K.V. 1964. Higher and specialized representatives of the ant genus Cataglyphis (Hymenoptera, Formicidae) in the fauna of the USSR. Zoologicheskii Zhurnal, 43 (12): 1800-1815. (in Russian)

ARNoldi K.V. 1967. New data on the ant genus Camponotus (Hymenoptera, Formicidae) from the USSR fauna. 1. Camponotus (s. str.). Zoologicheskii Zhurnal, 46 (4): 1815-1830. (in Russian)

ARNOLDI K.V. 1977. Review of the harvester ants of the genus Messor (Hymenoptera, Formicidae) in the fauna of the USSR. Zoologicheskii Zhurnal, 56 (11): 1637-1648. (in Russian)

Bolton B. 1994. Identification guide to the ant genera of the world. Harvard University Press, Cambridge, Massachusetts.

Bolton B. 2019. An online new general catalogue of the ants of the world, including a synopsis of taxonomic publications on Formicidae. Internet: http://antweb.org.1-3345. (Accessed 07 March 2018)

BorowiEC L., 2014. Catalogue of ants of Europe, the Mediterranean Basin and adjacent regions (Hymenoptera: Formicidae). Genus, 25 (1-2): 1-340.

BRULLÉ G.A. 1833. [1832] Expédition scientifique de Morée. Section des sciences physiques. Tome III. Partie 1. Zoologie. Deuxième section - Des animaux articulés. [part]. Levrault, Paris: 289-336. 
Collingwood C.A. 1985. Hymenoptera: Fam. Formicidae of Saudi Arabia. Fauna of Saudi Arabia, 7: $230-302$.

Collingwood C.A., Agosti D. 1996. Formicidae of Saudi Arabia. (Part 2). Fauna of Saudi Arabia, 15: $300-385$.

Csősz S., Heinze J., Mikó I. 2015. Taxonomic Synopsis Of The Ponto-Mediterranean Ants Of Temnothorax Nylanderi Species-Group. PloS One, 10 (11): e0140000.

Csősz S., Salata S., Borowiec, L. 2018. Three Turano-European species of the Temnothorax interruptus group (Hymenoptera: Formicidae) demonstrated by quantitative morphology. Myrmecological News, 26: 101-119.

DLuSSKY G.M., SoJunov O.S., ZABELIN S.I. 1990. Ants of Turkmenistan. Moscow University and Academy of Science of Turkmenistan, Ashabad.

DONISTHORPE H. 1946. A new subspecies of Messor FoREL, and a new variety of Aphaenogaster MAYR (Hym. Formicidae) from Turkey. Proceedings of the Royal Entomological Society of London. Series B, 15: 53-54.

EMERY C. 1925. Revision des espèces paléarctiques du genre Tapinoma. Revue Suisse de Zoologie, 32 (2): 45-64

Firouzi F., Pashaei Rad S., Hossein Nezhad Sh., Agosti D. 2011. Four new records of ants from Iran (Hymenoptera: Formicidae). Zoology in the Middle East, 52 (1): 71-78.

Forel A. 1886. Études myrmécologiques en 1886. Annales de la Société Entomologique de Belgique, 30: 131-215.

Forel A. 1889. Ameisen aus den Sporaden, den Cykladen und Griechenland, gesammelt 1887 von Herrn von OERTZEN. Berliner Entomologische Zeitschrift, 32 (2) [1888]: 255-265.

Forel A. 1894. Les Formicides de l'Empire des Indes \& de Ceylan. Part IV. Journal of the Bombay Natural History Society, 8: 396-420.

FOREL A. 1904. Dimorphisme du mâle chez les fourmis \& quelques autres notices myrmécologiques. Annales de la Société Entomologique de Belgique, 48: 421-425.

Forel A. 1910. Glanures myrmécologiques. Annales de la Société Entomologique de Belgique, 54: 6-32.

Ghahari H., Collingwood C.A., Tabari M., Ostovan, H. 2009. Faunistic notes on Formicidae (Insecta: Hymenoptera) of rice fields and surrounding grasslands of northern Iran. Munis Entomology \& Zoology, 4 (1): 184-189.

Ghahari H., Collingwood C.A., Havaskary M., Ostovan H., Samin N. 2011. A Contribution to the Knowledge of Ants (Hymenoptera: Formicidae) from the Arasbaran Biosphere Reserve and Vicinity, Northwestern Iran. Jordan Journal of Agricultural Sciences, 7 (3): 558-563.

Ghahari H., Sharaf M.R., Aldawood A.S., Collingwood C.A. 2015. A contribution to the study of the ant fauna (Hymenoptera: Formicidae) of Eastern Iran. Contribution to Entomology, 65 (2): $341-359$.

Ghobadi M., Agosti D., Mahdavi M., Jouri M.H., Majer J. 2016. Ants Visible from Space Influence Soil Properties and Vegetation in Steppe Rangelands of Iran. Sociobiology, 63 (4): 1063-1068. 
Heidari M.L., Zare Khormizi M., Moravvej Gh., Sadeghi Namaghi H. 2017. Survey on ants (Hymenoptera: Formicidae) and their aphid partners (Homoptera: Aphididae) in Northeast and Center of Iran. Entomofauna Zeitschrift für Entomologie, 38 (17): 369-376.

Hosseini A., Modarraes Awal M., Hosseini M. 2015. New faunistic records of Formicidae (Insecta: Hymenoptera) from Northeastern Iran. Asian Myrmecology, 7 (1): 113-127.

Hossein-Nezhad Sh., Pashaei Rad S., Firouzi F., Agosti D. 2012. New and additional records for the ant fauna from Iran. Zoology in the Middle East, 55 (1): 65-74.

KARAVAiEv V. 1924. Zur Systematik der paläarktischen Myrmecocystus (Formicidae), nebst einigen biologischen Notizen. Konowia, 3: 301-308.

Khandehroo F., Moravvej Gh., Sadeghi Namghi H. Fekrat L. 2015. New records of ant species (Hymenoptera: Formicidae) to the fauna of Iran: Camponotus alii Forel, 1890 and Proformica korbi (EMERY, 1909). Asian Myrmecology, 7: 129-131.

LAtreille P. A. 1798. Essai sur l'histoire des fourmis de la France. F. Bourdeaux, Brive.

KuZNETSOV-UGAmsKY N.N. 1927. Vorläufige Uebersicht über die mittelasiatischen Formen der Gattung Messor (Hym., Form.). Folia Myrmecologica et Termitologica, 1: 89-94.

KuZnetsov-Ugamsky N.N. 1929a. Die Gattung Acantholepis in Turkestan. Zoologischer Anzeiger, 82: 477-492.

KuZnetsov-Ugamsky N.N. 1929b. The ants of the genus Messor in the fauna of Middle Asia. Acta Universitatis Asiae Mediae. Series VIIIa Zoologia, 6: 1-25.

MAYR G. 1877. Formicidae. In: A.P. FedChEnko, Travels in Turkestan. Vol. 2, Div. 5, No. 7. Izvestiya Imperatorskago Obshchestva Lyubitelei Estestvoznaniya Antropologii i Etnografii pri Imperatorskom Moskovskom Universitete, 26: i-iii, 1-20 (+1).

Mirzamohamadi S., Hosseini M., Sadeghi Namaghi H., Karimi J., Mehrparvar M. 2015. Symbiotic ants (Hymenoptera: Formicidae) associated with aphids (Hemiptera: Aphididae) in Golestan province, Iran. Iranian Journal of Animal Biosystematics, 11 (2): 101-111.

Mohammadi S., Mossadegh M.S., Esfandiari M. 2012. Eight ant species (Hymenoptera: Formicidae) new for the fauna of Iran. Munis Entomology \& Zoology Journal, 7 (2): 847-851.

Moradloo Sh., Nafisi Fard R., Pashaei Rad Sh., Taylor B. 2015. Records of ants (Hymenoptera: Formicidae) from Northern Iran. Zoology in the Middle East, 61 (2): 168-173.

Mortazavi Z.S., Sadeghi H., Aktac N., Depa L., Fekrat L. 2015. Ants (Hymenoptera: Formicidae) and their aphid partners (Homoptera: Aphididae) in Mashhad region, Razavi Khorasan Province, with new records of aphids and ant species for Fauna of Iran. Halteres, 6: $4-12$.

Motschoulsky [ViCTOR, T.] 1839. Insectes du Caucase et des provinces transcaucasiennes. Bulletin de la Société Impériale des Naturalistes de Moscou, 12: 44-68.

PAKNIA O., KAMI H.G. 2007. New and additional records for the formicid fauna (Insecta: Hymenoptera) of Iran. Zoology in the Middle East, 40 (1): 85-90.

Paknia O., Radchenko A., Alipanah H., Pfeiffer M. 2008. A preliminary checklist of the ants (Hymenoptera: Formicidae) of Iran. Myrmecological News, 11: 151-59.

Paknia O., Radchenko A., Alipanah H., Pfeiffer M. 2010. New records of ants (Hymenoptera: Formicidae) from Iran. Asian Myrmecology, 3: 29-38. 
Pashaei Rad Sh., Taylor B., Torabi R., Aram E., Abolfathi G., Afshari R., Borjali F., Ghatel M., Hediary F., Jazini F., Heidary V., Mahmoudi Z., Safariyan F., Seiri M. 2018. Further records of ants (Hymenoptera: Formicidae) from Iran. Zoology in the Middle East, 64 (2): $145-159$.

PISARSKI B. 1965. Les fourmis du genre Cataglyphis FoERST. en Irak (Hymenoptera, Formicidae). Bulletin de l'Académie Polonaise des Sciences. Série des Sciences Biologiques, 13: 417-422.

PISARSKI B. 1971a. Nouvelles espèces de fourmis (Hymenoptera, Formicidae) du sous-genre Tanaemyrmex AsHM. d'Iraq. Bulletin de l'Académie Polonaise des Sciences. Série des Sciences Biologiques, 19: 671-675.

PISARSKI B. 1971b. Les fourmis du genre Camponotus MAYR (Hymenoptera, Formicidae) d'Iraq. Bulletin de l'Académie Polonaise des sciences. Série des sciences Biologiques, 19: 727-731.

RADChEnKo A.G. 1996a. Ants of the genus Plagiolepis MAYR (Hymenoptera, Formicidae) of central and southern Palearctic. Entomologicheskoe Obozrenie, 75 (1): 178-187. (in Russian)

RAdChEnKo A.G. 1996b. A key to the ant genus Camponotus (Hymenoptera, Formicidae) in Palearctic Asia. Zoologicheskii Zhurnal, 75: 1195-1203. (in Russian)

RADCHENKo, A.G. 1997. Review of ants of the subgenera Tanaemyrmex, Colobopsis, Myrmamblis, Myrmosericus, Orthonotomyrmex and Paramyrmamblis of the genus Camponotus (Hymenoptera, Formicidae) in the Asian Palearctic. Zoologicheskii Zhurnal 76: 806-815. (in Russian)

RADCHENKo A. 1998. Review of ants of the genus Cataglyphis FoERSTER (Hymenoptera, Formicidae) of Asia. Entomologicheskoye Obozreniye, 76 (2): 424-442. (in Russian)

Radchenko A., Paknia O. 2010. Two new species of the genus Cataglyphis Foerster, 1850 (Hymenoptera: Formicidae) from Iran. Annales Zoologici, 60 (1): 69-76.

Rafinejad J., Zareil A., Akbarzadeh K., Azad M., Biglaryan F., Doosti S., Sedaghat M.M. 2009. Faunistic study of ants with emphasis on the health risk of stinging ants in Qeshm Island, Iran. Iranian Journal of Arthropod-Borne Diseases, 3 (1): 53-59.

RuZSKY M. 1903. A new species of ant from the Transcaspian region. Russkoe Entomologicheskoe Obozrenie, 3: 36-37. (in Russian)

RUZSKY M. 1905. The ants of Russia. (Formicariae Imperii Rossici). Systematics, geography and data on the biology of Russian ants. Part I. Trudy Obshchestva Estestvoispytatelei pri Imperatorskom Kazanskom Universitete, 38: 1-800. (in Russian)

Salata S., Borowiec L., RadChenko A. 2018. Description of Plagiolepis perperamus, a new species from East-Mediterranean and redescription of Plagiolepis pallescens FOREL, 1889 (Hymenoptera: Formicidae). Annales Zoologici, 68 (4): 809-824.

SANTSCHI F. 1917. Acantholepis frauenfeldi MAYR, et ses variétés. Bulletin de la Société d'Histoire Naturelle de l'Afrique du Nord, 8: 42-48.

SANTSCHI F. 1927. Revision des Messor du groupe instabilis sm. (Hymenopt.). Boletín de la Real Sociedad Española de Historia Natural, 27: 225-250.

SEIFERT B. 2016. Inconvenient hyperdiversity - the traditional concept of "Pheidole pallidula" includes four cryptic species (Hymenoptera: Formicidae). Soil Organisms, 88 (1): 1-17.

SEIFert B., SChultz R. 2009. A taxonomic revision of the Formica rufibarbis FABRICIUS, 1793 group (Hymenoptera: Formicidae). Myrmecological News, 12: 255-272. 
Shiran E., Mossadegh M.S., Esfandiari, M. 2013. Mutualistic ants (Hymenoptera: Formicidae) associated with aphids in central and southwestern parts of Iran. Journal of Crop Protection, 2 (1): $1-12$.

Steiner F.M., Csösz S., Markó B., Gamisch A., Rinnhofer L., Folterbauer C., Hammerle S., Stauffer C., Arthofer W., Schlick-Steiner B.C. 2018. Turning one into five: integrative taxonomy uncovers complex evolution of cryptic species in the harvester ant Messor "structor". Molecular Phylogenetics and Evolution, 127: 387-404.

TонмÉ G. 1969. Description d'espèces nouvelles de fourmis au Liban (Hymenoptera Formicoidea). Publications de l'Université Libanaise. Section des Sciences Naturelles, 7: 1-15.

VAn LoOn A.J., BoOmsma J.J., AndRasfalvy A. 1990. A new polygynous Lasius species (Hymenoptera: Formicidae) from central Europe. I. Description and General Biology. Insectes Sociaux, 37 (4): 348-362.

Wagner H.C., Arthofer W., Seifert B., Muster C., Steiner F.M., Schlick-Steiner B.C. 2017. Light at the end of the tunnel: Integrative taxonomy delimits cryptic species in the Tetramorium caespitum complex (Hymenoptera: Formicidae). Myrmecological News, 25: 95-129.

WiLSON E.O. 1990. Success and Dominance in Ecosystems: The case of the Social Insects. Ecology Institute, Oldendorf/Luhe, Germany.

Received: 12 April 2019

Accepted: 28 April 2019 